\title{
Latest Higgs Physics results from the ATLAS experiment
}

\author{
Nicolo de Groot* \\ Radboud University and Nikhef \\ E-mail: N.deGroot@science.ru.nl
}

Since the discovery in 2012, many measurements have been made on the production and properties of the $125 \mathrm{GeV}$ Higgs boson. The latest result from ATLAS will be presented, including recent results from Run 1 at $8 \mathrm{TeV}$ and some obtained in Run 2 at $13 \mathrm{TeV}$.

Corfu Summer Institute 2016 "School and Workshops on Elementary Particle Physics and Gravity" 31 August - 23 September, 2016

Corfu, Greece

* Speaker.

${ }^{\dagger}$ On behalf of the ATLAS collaboration 


\section{Introduction}

The ATLAS and CMS Collaborations at the Large Hadron Collider (LHC) announced the discovery of a particle consistent with a Higgs boson in 2012 [1, 2]. Since then, the collaborations have together measured the mass of the particle to be about $125 \mathrm{GeV}$ [3]. Studies of its spin and parity in bosonic decays have found it to be compatible with a $J^{P}=0^{+}$state $[4,5]$. Combined coupling fits of the measured Higgs boson production and decay rates within the framework of the Standard Model (SM) have found no significant deviation from the SM expectations [6]. These results strongly suggest that the newly discovered particle is indeed a Higgs boson. In 2015 the LHC has been restarted with an increased center of mass energy of $\sqrt{s}=13 \mathrm{TeV}$. The higher crosssections and increasing luminosity allow the experiments to record larger samples of Higgs bosons and study their properties in greater detail.

At the LHC, SM production of the Higgs boson is dominated by the gluon fusion process $g g \rightarrow$ $H(\mathrm{ggF})$, followed by the vector-boson fusion process $q q^{\prime} \rightarrow q q^{\prime} H$ (VBF). Associated production with a W boson $q \bar{q} \rightarrow W H(\mathrm{WH})$, a $\mathrm{Z}$ boson $q \bar{q} / g g \rightarrow Z H(\mathrm{ZH})$ or with a pair of top quarks $q \bar{q} / g g \rightarrow t \bar{t} H(\mathrm{ttH})$ have sizeable contributions as well. The $\mathrm{WH}$ and $\mathrm{ZH}$ production processes are collectively referred to as the $\mathrm{VH}$ process.

ATLAS [7] is a general-purpose detector designed for a broad programme of particle physics measurements. An inner tracking detector, located within a $2 \mathrm{~T}$ axial magnetic field generated by a superconducting solenoid, is used to measure the trajectories and momenta of charged particles. The innermost layers consisting of high-granularity silicon pixel detectors instrument a pseudorapidity range $|\eta|<2.5$. A new innermost silicon pixel layer, the Insertable B-Layer [8] (IBL), was added to the detector between Run 1 and Run 2. The IBL improves the experiment's ability to identify displaced vertices and thereby significantly improves the b-tagging performance. Beyond the pixel detectors there are silicon strip detectors with coverage within $|\eta|<2.5$. Outside this, covering $|\eta|<2.0$ additional straw tube tracking detectors are placed. These provide measurements of transition radiation that contribute to the identification of electrons. The calorimeter system consists of a number of different technologies with coverage out to $|\eta|<4$.9. The liquid-argon electromagnetic calorimeter is divided into three regions: barrel and two endcaps . The hadronic calorimetry surrounds the electromagnetic calorimeters, using scintillator tiles or liquid argon as the active materials. The outermost layers of the detector are taken up by the muon spectrometer that measures the trajectories of muons bending in the field of three large air-core toroidal magnets. High precision tracking can be achieved out to $|\eta|<2.7$ and there are additional chambers for fast triggering within the range $|\eta|<2.4$. A two-level trigger system [9] is used to reduce the recorded data rate from $40 \mathrm{MHz}$ to a level of around $1 \mathrm{kHz}$. The first level is a hardware implementation that makes use of only a subset of the total available information to make fast decisions to accept or reject an event, aiming to reduce the rate to around $100 \mathrm{kHz}$, and the second level is the software based High-Level Trigger that provides the remaining rate reduction.

\section{Higgs decay to bosons}

The Higgs boson decay into two photons $H \rightarrow \gamma \gamma$ is a particularly attractive way to study the properties of the Higgs boson. Despite the small branching ratio, a reasonably large signal yield 
can be obtained thanks to the high photon reconstruction and identification efficiency at ATLAS. Furthermore, due to the excellent photon energy resolution of the ATLAS calorimeter, the signal manifests itself as a narrow peak in the diphoton invariant mass spectrum on top of a smoothly falling background, and the Higgs boson signal yield can be measured using an appropriate fit as is shown in Figure 1. The Higgs decay into a pair of photons was one of the discovery channels of Run 1. A new Run 2 result [10] is presented using $13.3 \mathrm{fb}^{-1}$ of data.

The fiducial cross section is measured to be $\sigma_{\text {fid }}=43.2 \pm 14.9$ (stat.) \pm 4.9 (syst.) fb for a Higgs boson of mass $125.09 \pm 0.21$ (stat) \pm 0.11 (syst) $\mathrm{GeV}$, decaying to two isolated photons that have transverse momentum greater than $35 \%$ and $25 \%$ of the diphoton invariant mass and each with absolute pseudorapidity $|\eta|<2.37$, excluding the region $1.37<|\eta|<1.521$. The Standard Model prediction for the same fiducial region is $62.8_{-4.4}^{+3.4} \mathrm{fb}$.

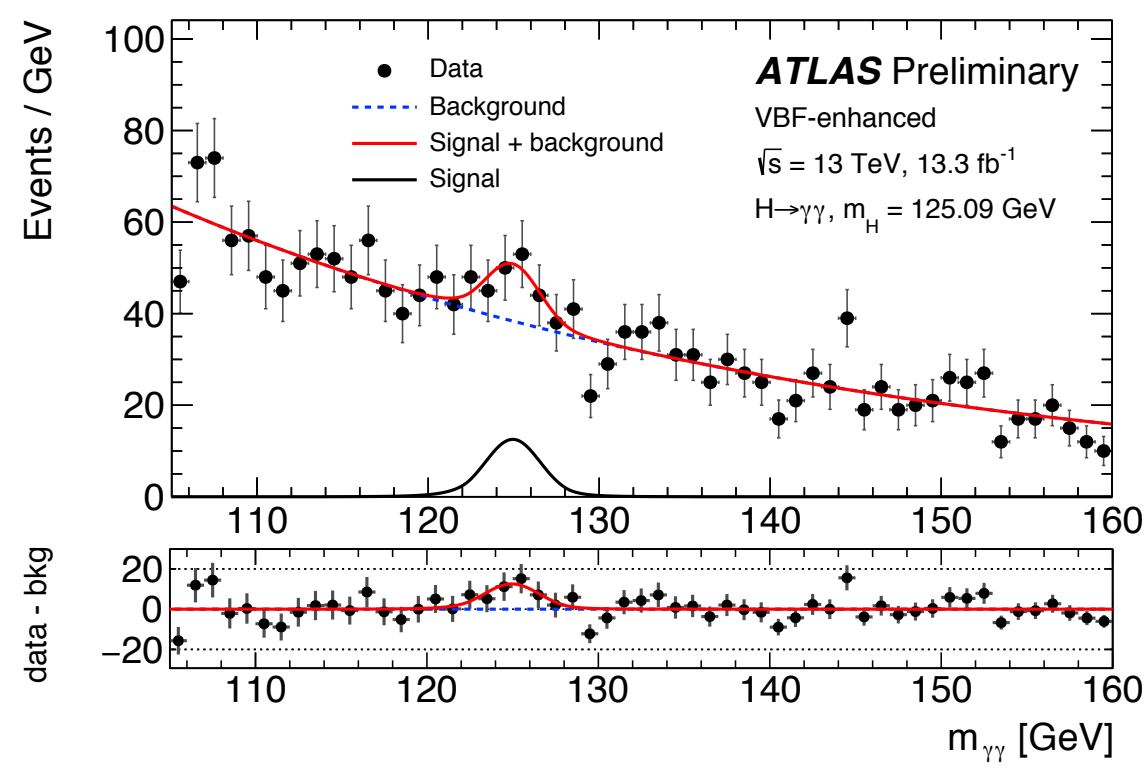

Figure 1: Invariant mass of the photon pair for the vector boson fusion sample [10]

Differential cross-sections as a function of jet multiplicity, jet transverse momentum, the angle between the beam axis and the photons and the angular separation between the jets in the Higgs + 2 jets sample have also been measured and compared to theoretical prediction.

The other discovery channel has been the $Z Z^{*}$ with 4 leptons in the final state. This is the cleanest decay mode with only little background from diboson production and $Z+$ jets. In the new Run 2 analysis [11] the properties of the Higgs boson decaying to four leptons are studied using $14.8 \mathrm{fb}^{-1}$ of integrated luminosity. The results include measurements of the fiducial cross sections, the different production mode cross sections and a test of beyond the Standard Model interactions using an effective Lagrangian approach. The signal contribution is extracted from the 4-lepton invariant mass spectrum, which can be seen in Figure 2.

The results for $\gamma \gamma$ and $Z Z^{*}$ are combined in [12]. Results are derived for these two decay modes and five sets of production processes for a Higgs boson rapidity $\left|y_{H}\right|<2.5$ and for the same Higgs boson mass of $125.09 \mathrm{GeV}$. The global signal strength, defined as the ratio in the 


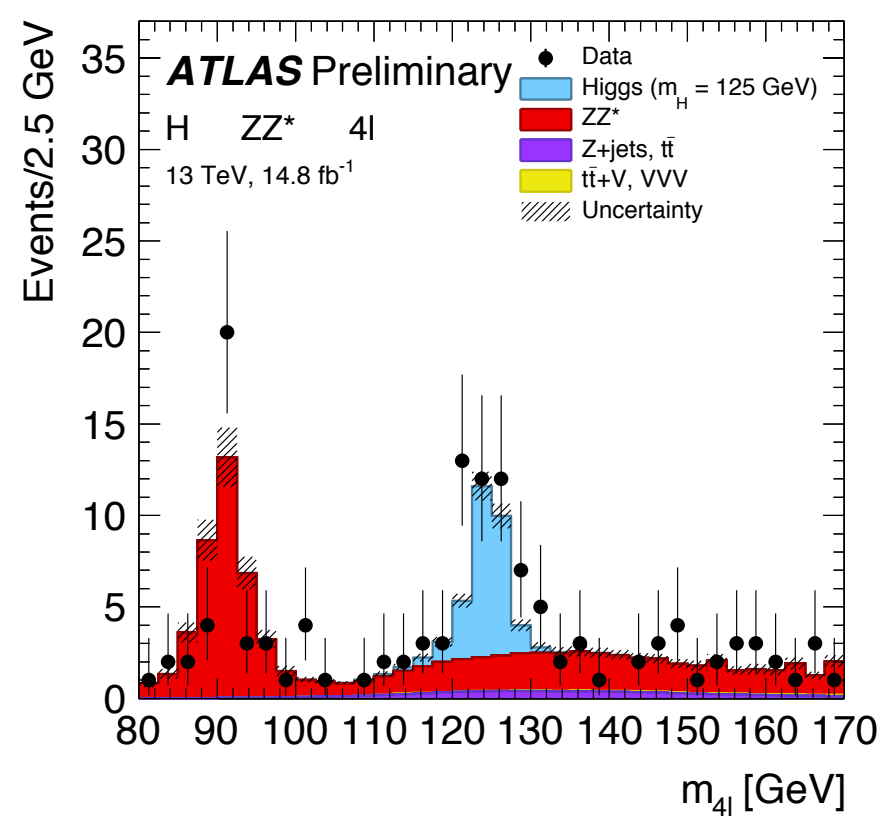

Figure 2: The 4 lepton invariant mass spectrum [11]

full phase space (including $\left|y_{H}\right| \geq 2.5$ ) of the observed total signal yield and the Standard Model expectation, is measured to be $\mu=1.13_{-0.17}^{+0.18}$. The cross section of $p p \rightarrow H+X$ in the full phase space is determined from fiducial cross section measurements to be $59.0_{-9.2}^{+9.7}$ (stat. $)_{-3.5}^{+4.4}$ (syst.) pb, to be compared with the Standard Model prediction of $55.5_{-3.4}^{+2.4} \mathrm{pb}$. This result is shown together with those of Run 1 compared to the theory predictions in Figure 3. No significant deviation from the Standard Model expectations is observed.

\section{Higgs and top quarks}

A measurement of Higgs production in association with a top quark provides a direct probe of the $t t H$ Yukawa coupling as opposed to the measurement of the gluon-gluon fusion production cross-section, which probes this same coupling in a top quark loop. Any significant discrepancy between the two could indicate the presence of new physics in the loop. The $t t H$ production process benefits significantly from the higher beam energy in Run 2. The Standard Model cross-section for this process is a factor 4 higher than in Run 1 . Several analyses using $13.2 \mathrm{fb}^{-1}$ of Run 2 data are presented here.

A search for $t t H$ production with the Higgs boson subsequently decaying into a pair of $b$ quarks has been performed [13]. The search uses events with at least one top quark decaying to an electron or muon. Events are categorized according to the jet multiplicity and the number of jets containing a b-hadron decay, and multivariate techniques are used to discriminate between signal and background events, the latter being dominated by $t \bar{t}+$ jets production. The categories with a high number of jets and $b$ jets have the best signal over background ratio and are used as signal regions. For the one lepton case for instance, these are the 6 jet 4 b, 6 jet 3 b and 5 jet 3 


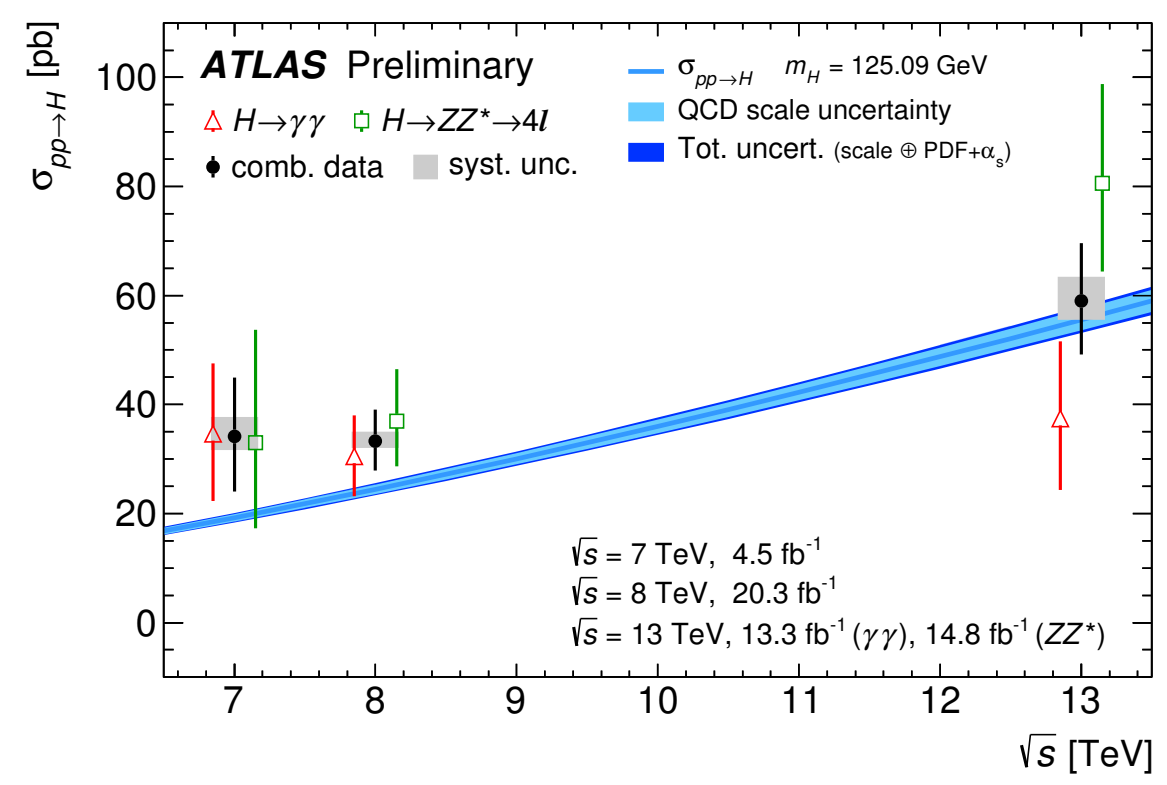

Figure 3: The Higgs production cross-section for the $\gamma \gamma$ and $Z Z^{*}$ decay mode as a function of the center of mass energy [12].

b categories. The other categories are used as control regions to constrain the background. The data are consistent with either the background-only hypothesis or with the Standard Model $t t H$ prediction. The ratio of the measured $t t H$ signal cross-section to the Standard Model expectation is found to be $\mu=2.1_{-0.9}^{+1.0}$, assuming a Higgs boson mass of $125 \mathrm{GeV}$. The dominant error for this analysis is the modeling of the $t \bar{t}+$ heavy flavor background.

Another analysis [14] is targeting final state with multiple leptons which originate predominantly from Higgs decaying into a pair of $\mathrm{W}$ bosons or a pair of $\tau$ leptons. The best-fit value of the ratio of observed and Standard Model cross sections is $\mu=2.5 \pm 0.7$ (stat) ${ }_{-0.9}^{+1.1}$ (syst), and an upper limit on this ratio of 4.9 (2.3 expected) is found at $95 \%$ confidence level.

The results of the two analyses are combined with the result for $t t H$ with Higgs decaying into two photons from [10] to extract an average signal strength for $t t H$ [15]. The results are shown in Figure 4. The ratio of the production cross section extracted from the data, assuming the Standard Model Higgs boson decay pattern, and the corresponding Standard Model prediction is found to be $\mu=1.8 \pm 0.7$. This corresponds to an observed significance of $2.8 \sigma$, where $1.8 \sigma$ would be expected for Standard Model $t t H$ production. The sensitivity of this combination exceeds the Run 1 expected significance of $1.5 \sigma$.

\section{VH and VBF Higgs production to $b$ quarks}

The Higgs decay to $\mathrm{b}$ quarks benefits from the largest branching fraction of $58 \%$. Unfortunately the gluon-gluon fusion process is swamped by the QCD background which is many orders of magnitude larger and something is needed to tag the Higgs production and suppress the background. 


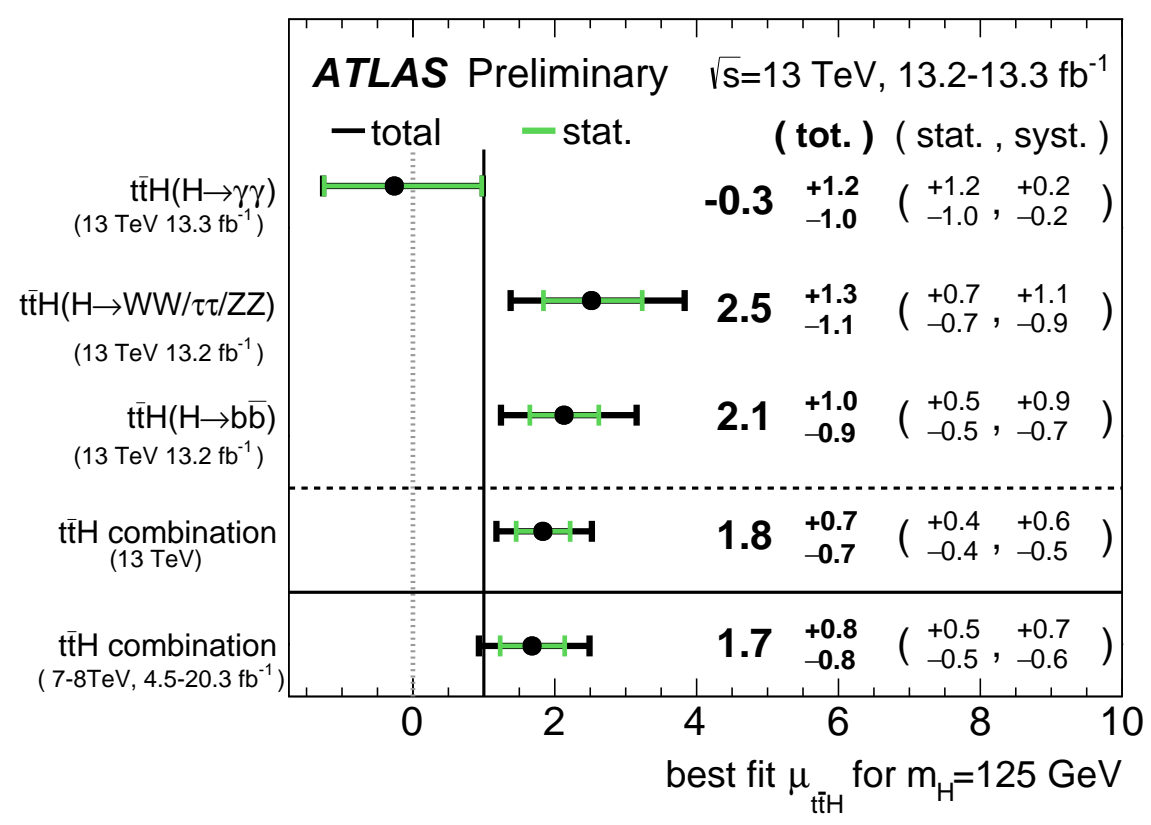

Figure 4: Combination of the various decay modes in the ttH channel [15]

One possibility is to use Higgs production in association with a vector boson as in [16], an analysis which uses $13.2 \mathrm{fb}^{-1}$ of data from Run 2. The analysis distinguishes final states with 0 , 1 or 2 leptons, with the 1-lepton state coming dominantly from WH production and the 2-lepton state from $\mathrm{ZH}$ production. In the case of the $Z$ decaying to neutrinos, the final state will have 0 leptons in combination with substantial missing energy. The analysis uses multivariate techniques to fight the backgrounds, which are dominated by top pair production and $Z b b$ production. The best discrimination is obtained from the invariant mass distribution of the $\mathrm{b}$ jets, $m_{b b}$ and from the angular separation of the these jets. Figure 5 summarizes the results. In none of the channels we observe a clear signal.

In vector boson fusion some background suppression is found in the presence of the forward tagging jets and the rapidity gap between them. An analysis using the full Run 1 dataset [17] has been performed. This analysis is using boosted decision trees to fight the QCD and Zbb backgrounds and fits the $m_{b b}$ spectrum to extract the signal. No signal is observed and a 95\% CL limit is found of 4.4 times the Standard Model cross-section, consistent with the expected limit of 5.7 times the SM production hypothesis.

The VBF channel can be further cleaned-up by requiring an additional photon in the final state. The search in the $b \bar{b} \gamma j j$ signature benefits from a large reduction of QCD jet background relative to the inclusive $b \bar{b} j j$ signature and from the presence of a high tranverse-momentum photon for triggering. Results are reported from the analysis of $12.6 \mathrm{fb}^{-1}$ Run 2 data. The observed (expected) 95\% confidence level upper limit on the production cross section times branching ratio for a Higgs mass of $125 \mathrm{GeV}$ is 4.0 (6.0) times the Standard Model expectation. Figure 6b shows some of the leading diagrams for both analyses. 


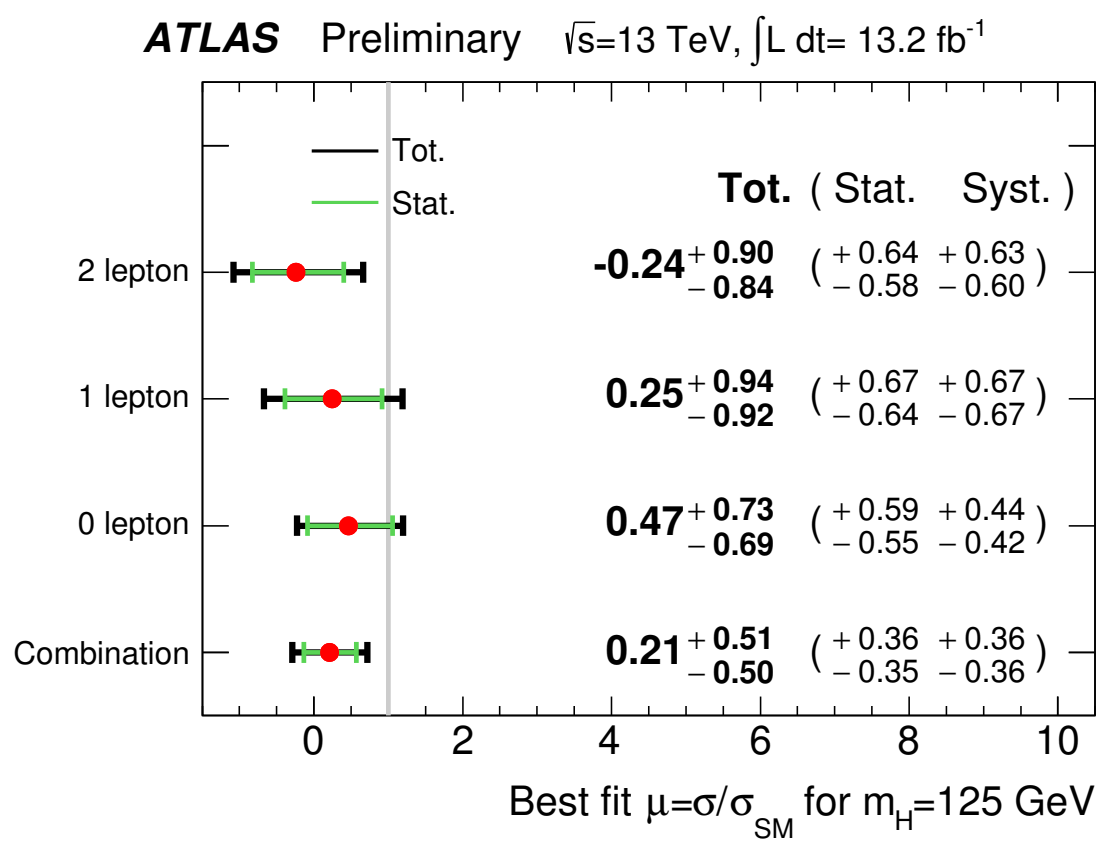

Figure 5: Ratios of the measured cross-sections of VH production divided by the Standard Model value [16]

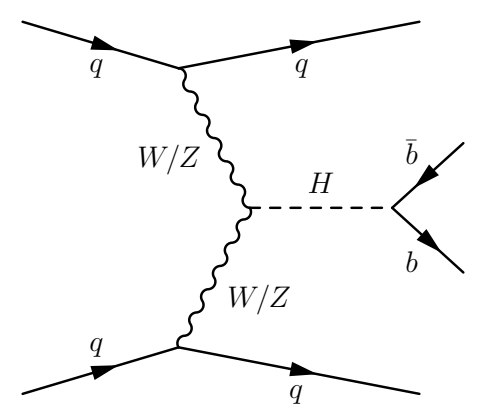

(a)

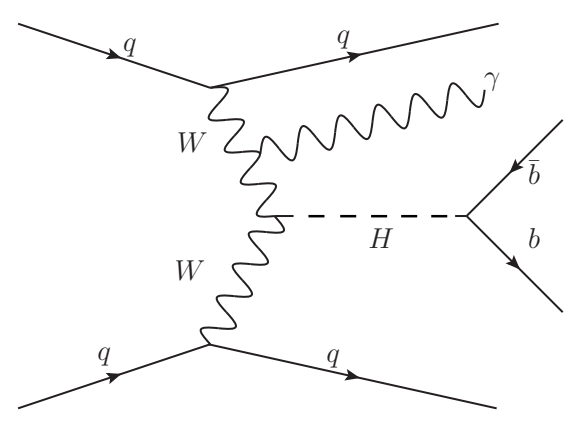

(b)

Figure 6: Diagram for the VBF process (a) and with an additional photon (b)

\section{Rare and forbidden decays}

The decay of the Higgs boson into a pair of muons is a rare decay with a branching ratio in the Standard Model of $2 \times 10^{-4}$. It is interesting for a number of reasons. It would be the first probe of the Higgs boson coupling to fermions of the second generation. It would also show the Higgs mechanism at work at a mass scale which is two orders of magnitude lower than what we have measured so far. An analysis [19] has used $13.2 \mathrm{fb}^{-1}$ of Run 2 data to look for the signature of this decay. The dominant background is coming from $Z$ decaying to a muon pair. This background is less severe in the Vector Boson Fusion Channel, which is treated separately. Figure 7 is showing the invariant mass of the muon pairs for the VBF together with the projected signal. The analysis finds a measured (expected) 95\% CL limit of 4.4 (5.5) times the SM value. Together with the Run 1 


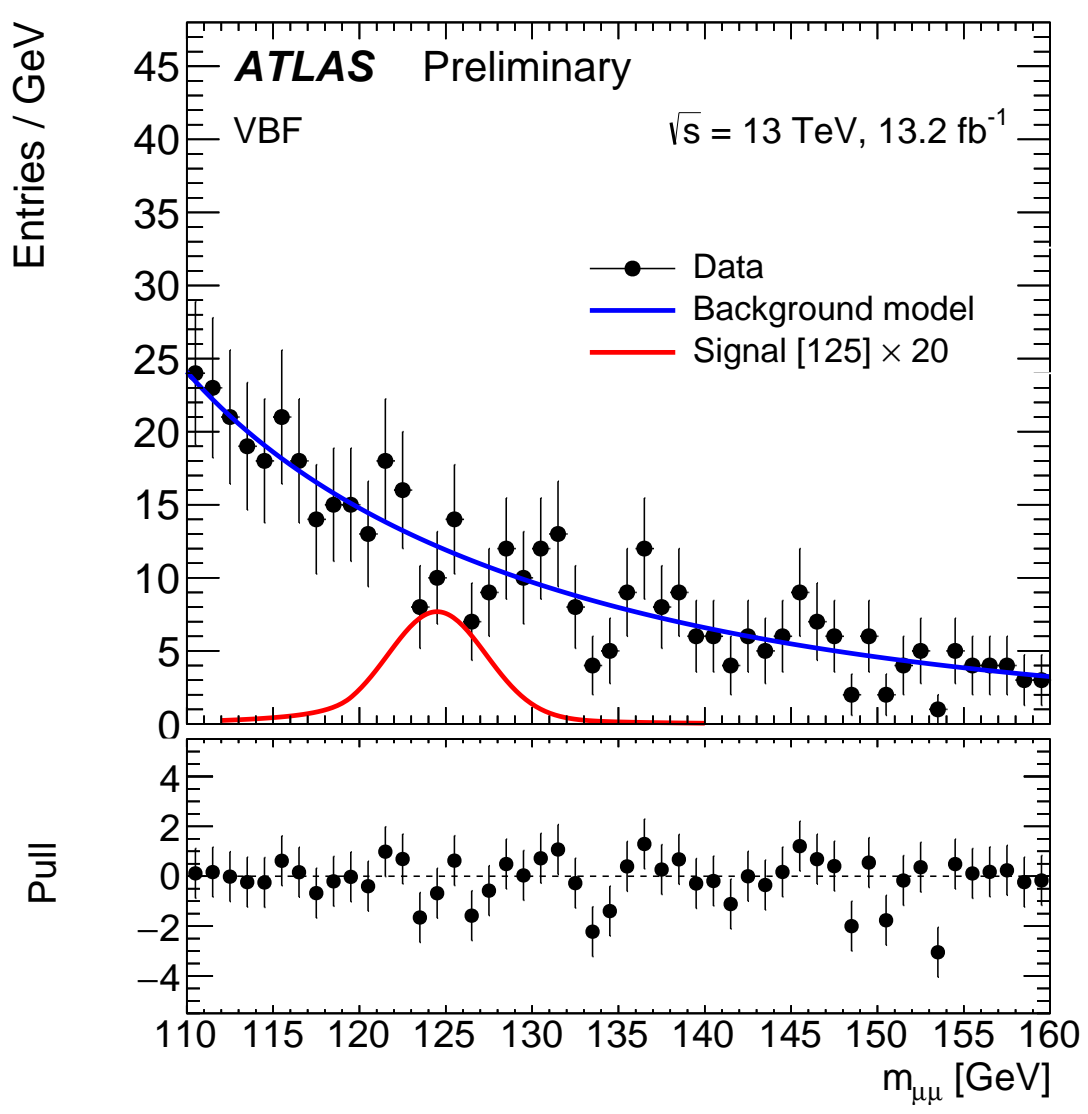

Figure 7: The invariant $\mu \mu$ mass for the most sensitive VBF category [19]

result the limit is 3.5 (4.5) times the SM value.

ATLAS has performed a search for lepton flavor violating decays of the Higgs boson [20]. The analysis looks for the decay of a Higgs boson in one hadronic tau and one muon or electron. The Higgs candidate mass is reconstructed using the missing mass calculator [21] to recover the energy of the neutrinos in the tau decay. Figure 8 shows the $M M C$ mass in the $\mu \tau$ channel for the major backgrounds and the projected signal. No signal was observed. The analysis puts a limit on the branching ratio of the decay $H \rightarrow e \tau$ of $1.0 \%$ and $H \rightarrow \mu \tau$ of $1.4 \%$.

\section{Conclusion}

The LHC has made an impressive start and the Higgs samples collected by the ATLAS experiment are already matching or exceeding those of Run 1 . Several results have been presented in this paper, and we can expect many more. Next to measurements of Higgs coupling, we are also preparing studies of differential distributions and CP-violation and to use the Higgs boson as a probe for new physics. Run 2 will continue until 2018 and should deliver a Higgs sample which will be an order of magnitude larger than that of Run 1. Higgs physics will become precision Standard Model physics. 


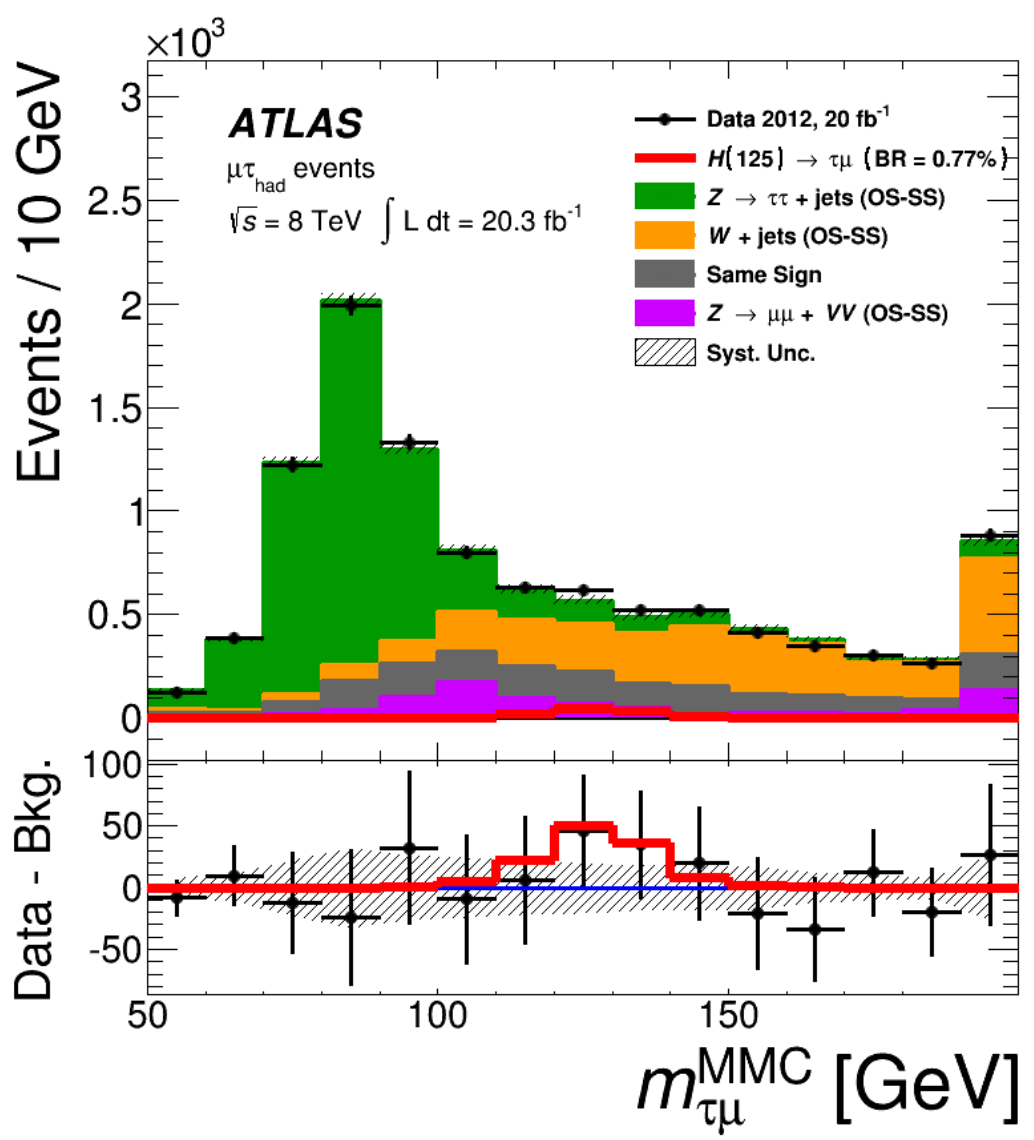

Figure 8: The invariant $\mu-\tau$ mass calculated from the Missing Mass Calculator for the Signal Regions [20]

\section{References}

[1] ATLAS Collaboration, Phys. Lett. B716 (2012) 1.

[2] CMS Collaboration, Phys. Lett. B716 (2012) 30.

[3] ATLAS and CMS Collaborations, Phys. Rev. Lett. 114 (2015) 191803

[4] ATLAS Collaboration, Eur. Phys. J. C75 (2015) 476.

[5] CMS Collaboration Phys. Rev. D 92 (2015) 012004

[6] ATLAS and CMS Collaborations, JHEP 1608 (2016) 045.

[7] ATLAS Collaboration, 2008 JINST 3 S08003.

[8] ATLAS Collaboration, ATLAS-LHCC-2010-013 (2010), http://cdsweb.cern.ch/record/1291633

[9] ATLAS Collaboration, ATLAS-DAQ-PUB-2016-001 (2016), http://cdsweb.cern.ch/record/2136007

[10] ATLAS Collaboration, ATLAS-CONF-2016-067, http://cdsweb.cern.ch/record/2206210

[11] ATLAS Collaboration, ATLAS-CONF-2016-079, http://cdsweb.cern.ch/record/2206253

[12] ATLAS Collaboration, ATLAS-CONF-2016-081, http://cdsweb.cern.ch/record/2206272 
[13] ATLAS Collaboration, ATLAS-CONF-2016-080, http://cdsweb.cern.ch/record/2206255

[14] ATLAS Collaboration, ATLAS-CONF-2016-058, http://cdsweb.cern.ch/record/2206153

[15] ATLAS Collaboration, ATLAS-CONF-2016-068, http://cdsweb.cern.ch/record/2206211

[16] ATLAS Collaboration, ATLAS-CONF-2016-091, http://cdsweb.cern.ch/record/2206813

[17] ATLAS Collaboration, JHEP 11 (2016) 112

[18] ATLAS Collaboration, ATLAS-CONF-2016-063, http://cdsweb.cern.ch/record/2206201

[19] ATLAS Collaboration, ATLAS-CONF-2016-041, http://cdsweb.cern.ch/record/2206079

[20] ATLAS Collaboration, JHEP 11 (2015) 211.

[21] A. Elagin et al., Nucl. Instrum. Meth. A 654 (2011), 